\title{
Imaginäre Grenzen. Zur Entstehung impliziter Kollektivität in EU-Europa
}

\author{
Andreas Langenohl
}

Zusammenfassung: Der Aufsatz untersucht mittels einer praxistheoretischen Agenda die Konstruktion sozialer, ökonomischer und kultureller Grenzlinien innerhalb der Europäischen Union. Empirisch wird das Fallbeispiel von Interaktionsformen, Zirkulationspraktiken und öffentlichen Diskursen im Rahmen eines Städtepartnerschaftsrings in einer Kleinstadt untersucht. Als theoretische Grundposition fungiert der Begriff des „,social imaginary“, der von Charles Taylor und anderen in die Diskussion gebracht wurde. Er besagt, dass soziale „Bedeutung“ - etwa hinsichtlich der Grenzen zwischen Gruppen, Ländern, Schichten etc. - oftmals nicht intendiert und explizit „konstruiert“ wird, sondern gleichsam als Nebenprodukt sozialen Praktiken entsteigt, die nicht in erster Linie auf die Produktion jener „Bedeutung“ hin entworfen sind. Im Falle von Städtepartnerschaften sind dies in erster Linie Praktiken der Zirkulation von Waren und Personen, die auf ihre Grenzziehungsimplikationen hin zu analysieren sind. Abschließend wird das spezifisch Europäische dieser imaginären Grenzen herausgearbeitet: Die praktischen Grenzziehungen und Grenzbedeutungen, die durch Aktivitäten von Städtepartnerschaftsorganisationen hervorgebracht werden, stehen in einer Kontinuität mit dem politischen Projekt der Europäischen Union, weil sie auf ein Paradigma politischer Integration bezogen sind, das der Ökonomie eine gewisse Dignität zugesteht und die EU als Ganze prägt.

Schlüsselwörter: Europäische Union · Grenze · Imaginäres · Identität · Markt

Imaginary borders: The emergence of implicit collectivities in EU-Europe

Abstract: Drawing on a practice-theoretical approach inspired by the notion of the social imaginary, imaginary borders in the European Union are conceptualized as the public emergence, maintenance and modification of interpretations about European borders through practices of circulation of symbols, commodities, and people. The construction of borders in the EU is thus neither located on the attitudinal level nor conceived of as the deliberate construction of meaning, but as the unintended emergence of the border as a meaningful category from taken-for-granted practices carrying their own meaning. Focusing on twin towns' activities in the European Union as a paradigmatic location for the articulation of political-cultural understandings of the EU, it is argued that these articulations are not only embedded within economic and social circuits, but in the first place

PD Dr. A. Langenohl (ه)

Cluster EXC16 „Kulturelle Grundlagen von Integration“, Universităt Konstanz, Fach 211,

78457 Konstanz, Deutschland

E-Mail: andreas.langenohl@uni-konstanz.de 
emerge from them as taken-for-granted and imaginary meanings of what the EU as a polity is and where its borders are. Methodologically, this calls for an understanding of the relationship between imaginary political collectivity and its economic, social and cultural channels of circulation which is not conceived as a text-context-relation but as one of mutual imaginary constitution.

Keywords: European Union · Border · Imaginary $\cdot$ Identity $\cdot$ Market

\section{Frontières imaginaires. Sur l'émergence d'une collectivité implicite dans l'Union européenne}

Résumé: Cet article étudie la construction des frontières sociales, économiques et culturelles au sein de l'Union européenne sous l'angle d'une théorie de la pratique. Concrètement, il s'agit d'une analyse de l'exemple des formes d'interaction, des pratiques de circulation et des discours publics dans le cadre du cercle de jumelage d'une petite ville. La position théorique adoptée est basée sur le concept d',,imaginaire social" proposé entre autres par Charles Taylor. Ce concept est sous-tendu pas l'idée que, bien souvent, la ,signification“ sociale - par exemple des frontières entre groupes, pays, couches sociales, etc. - ne relève pas d'une intention et d'une „,construction“ explicite mais surgit en quelque sorte comme un sous-produit de pratiques sociales ne visant pas en premier lieu à produire cette „signification“. Dans le cas des jumelages, il s'agit en premier lieu d'analyser les pratiques de circulation des marchandises et des personnes sous l'angle de leurs implications en terme de traçage de frontières. En conclusion, nous faisons ressortir ce que ces frontières imaginaires ont de spécifiquement européen: le traçage et la signification pratique des frontières résultant des activités des comités de jumelage s'inscrivent dans le projet politique de l'Union européenne en ceci qu'ils renvoient à un paradigme d'intégration politique qui accorde à l'économie une certaine dignité et imprime sa marque à l'UE dans son ensemble.

Mots-clés: Union européenne $\cdot$ Frontière $\cdot$ Imaginaire $\cdot$ Identité $\cdot$ Marché

\section{Einleitung}

Als Projektionsfläche für Theorien politischer, sozialer und kultureller Integration und Grenzziehungen hat Europa schon längere Zeit Konjunktur (Beck u. Delanty 2006; Delanty 2006; Habermas 2001; Krossa 2009; Meyer 2004). Die empirische „Europasoziologie" (Vobruba 2008) steckt hingegen noch in den Kinderschuhen. Der vorliegende Aufsatz soll hierzu einen Beitrag leisten. Georg Vobruba (ebd.) hat angemerkt, dass das Betätigungsfeld der Europasoziologie aus Spannungen erwachse, die zwischen den institutionellen Strukturen und Grenzen der Europäischen Union einerseits und Wahrnehmungen verschiedener Akteursgruppen andererseits erwüchsen. Grenzen seien daher nicht als institutionell gegeben zu betrachten, sondern als Deutungsleistungen, die wohl auf die europäischen Institutionen Bezug nähmen, sie aber je unterschiedlich interpretierten und werteten. Daraus ergibt sich das allgemeine Argument, dass institutionelle Strukturen und Grenzen und die Ressourcen, die sie zur Verfügung stellen, nicht identisch sind mit den „Grenzen in den Köpfen".

An dieser Unterscheidung setzt der vorliegende Aufsatz an, fasst sie allerdings weniger als eine empirisch gegebene denn als eine analytische Unterscheidung auf. Gerade weil institutionelle Strukturen und ,mental maps" oftmals nicht übereinstimmen, ist es erforderlich, die Frage zu stellen, in welchem Verhältnis sie zueinander stehen und wie 
man dieses Verhältnis theoretisch und methodologisch fassen kann. Auf eine solche Frage versprechen praxistheoretische Ansätze Antworten. Der vorliegende Artikel stellt daher zunächst einen theoretischen Bezug zwischen Praktiken im Kontext institutionalisierter Strukturen in der EU (nicht der „Brüsseler“, sondern zivilgesellschaftlicher Institutionen) und kulturellen Rahmungen und Grenzziehungen her. Er knüpft dabei an unlängst erschienene soziologische Arbeiten zu transnationaler Kommunikation in Europa ebenso an wie an theoretische Modellierungen der Praxisverankerung kultureller Rahmungen, die mit dem Begriff des „Imaginären“" operieren.

Daran schließt sich eine empirische, ethnografisch wie auch inhaltsanalytisch vorgehende Untersuchung an, die dem Zusammenhang zwischen europäischen Praktiken und Konstruktionen der (Binnen-)Grenzen Europas am Beispiel von Städtepartnerschaften nachgeht. Städtepartnerschaften, obwohl in der Forschung deutlich unterrepräsentiert, sind auf Europa und die Europäische Union in vielfältiger Weise bezogen: historisch, weil sie nach dem Zweiten Weltkrieg ins Leben gerufen wurden, um die ehemaligen Kriegsgegner zu versöhnen; institutionell, weil Städtepartnerschaften bzw. ihre Organisationen in den allermeisten Fällen von der Europäischen Union finanziert werden und über ihre Aktivitäten Rechenschaft ablegen; programmatisch, weil es zu ihren selbst gesetzten und den von ihnen erwarteten Aufgaben gehört, Europa stärker in den „Köpfen der Menschen" zu verankern; paradigmatisch, weil Städtepartnerschaften zu den Organisationsformen gehören, die einer „,europäischen Aktivbürgerschaft" (Wiesner 2007) am nächsten kommen. Die Fallstudie richtet sich daher auf den empirischen Zusammenhang zwischen Praktiken eines Städtepartnerschaftsrings und den imaginären Grenzkonstruktionen Europas, die diesen Praktiken gleichsam entsteigen. Das Hauptergebnis sei vorweggenommen - die imaginären Grenzen EU-Europas werden in Begriffen marktförmigen Austauschs gerahmt, das heißt der legitime Zugang zur EU wird mit dem Zugang zu einem Gemeinsamen Markt gleichgesetzt, der die EU imaginär repräsentiert. Am Ende wird gefragt, worin die europäische Spezifik der untersuchten Praktiken und kulturellen Grenzkonstruktionen besteht (Abschnitt 6).

\section{Das Problem der „Transzendierung“ von Zweckgemeinschaften in Europa}

Der Debatte um die Grenzen Europas hat sich die empirisch vorgehende Soziologie erst vor Kurzem zugewandt. Diese Debatte wurde seit den 1990er Jahren von zwei Positionen dominiert, die nach den kulturellen und normativen Grundlagen der Europäischen Union als ein politisches Projekt fragten. Die Debatte wogte über einen ausgedehnten Zeitraum zwischen Europaskeptikern und Europabefürwortern hin und her: Während die Europaskeptiker anführten, dass die EU niemals ein politisches Gemeinwesen sein könne, weil ihm ein kulturell verankerter „demos“ fehle (die sogenannte „no demos“-These, vgl. Kielmansegg 1996; Böckenförde 1997; Greven 1998), argumentierten die Befürworter, dass ein solcher „demos" erst als Folge politischer Integration überhaupt entstehe und daher nicht essenzialistisch vorausgesetzt werden müsse (Habermas 1998, 2001; ReeseSchäfer 1999; Lepsius 1999; Meyer 2004; vgl. auch Wiesner 2007).

Ein Effekt dieser Auseinandersetzung ist gewesen, dass sich empirisch vorgehende Analysen, die nicht im politisch-theoretischen Register dieser Debatte argumentierten, 
erst eine Stimme erkämpfen mussten. Dieser Moment kann mit der Geburtsstunde einer genuinen „Europasoziologie“ (Vobruba 2008, S. 32) gleichgesetzt werden, da nun Fragen bearbeitbar wurden, die sich nicht auf die politisch-theoretischen Bedingungen von Identifikationen und Grenzsetzungen in der EU, sondern auf letztere selbst bezogen.

Diese europasoziologischen Arbeiten müssen indes sorgfältig von bereits seit einiger Zeit durchgeführten Studien unterschieden werden, die den Prozess der europäischen Integration auf nationalstaatlicher Ebene untersuchten, etwa vergleichende Umfragestudien zur Identifikationsbereitschaft der Bürger mit der Europäischen Union in den einzelnen Mitgliedsstaaten (Immerfall u. Sobisch 1997; Fuchs u. Klingemann 2000; Gerhards 2004) oder zur wechselseitigen Abgrenzung und Identifikation der europäischen Nationalbevölkerungen von- bzw. untereinander (vgl. zusammenfassend Vobruba 2008, S. 42 f.). Gegenüber diesen Studien sind methodologische Vorwürfe erhoben worden: Die Messung von Einstellungen operiere mit Instrumenten, die in historisch westlichen Gesellschaften entwickelt worden seien und daher zu einem westeuropäischen „bias“ tendierten, der andere Gesellschaften benachteilige; die Legitimität supranationaler politischer Gebilde lasse sich nicht in nationalstaatlichen kognitiven Rahmungen messen und vergleichen (Soysa u. Zervakis 2002; Trenz et al. 2003); es werde mit am Nationalstaat entwickelten Instrumenten etwas gemessen, was im Vergleich zum Nationalstaat und der Nation wesentlich jünger sei und daher nicht im selben Maße in den Daten präsent sein könne; und die kulturellen Rahmungen, die politische Wahrnehmungen bestimmten, seien nicht auf der Einstellungsebene, sondern auf der interindividuellen Ebene als ,,kollektiv geteilter Sinnhorizont" angesiedelt (Eder 2007, S. 46).

Auf diese Vorwürfe reagiert die Europasoziologie in erster Linie mit einem heuristischen Fokus, der nicht nach nationalstaatlich institutionalisierten oder gerahmten Bedingungen der Identifikation mit Europa fragt, sondern faktisch existierende, transnationale Kommunikationskanäle, soziale Praktiken, Netzwerke und Sinnhorizonte auf europäischer Ebene empirisch untersucht. Diese Forschung nahm ihren Anfang naheliegender Weise bei den Medien der Massenberichterstattung, in denen über die politische Verfasstheit der EU diskutiert wird. Hier lag die Evidenz transnationaler Bezugnahmen und Identifikationen in Europa, nämlich in Form von Verweisen der Debatte über nationalstaatliche und -sprachliche Grenzen hinaus, auf der Hand (Eder et al. 2000; Eder 2003; Steeg 2003; Trenz 2001). In diesen neueren Studien werden massenmediale Manifestationen politischer Kultur untersucht bzw. die Trajekte von Bedeutungseinheiten, ihre Übersetzungen in andere Kontexte (beispielsweise andere Nationalsprachen und nationale Massenmedien) und die daraus resultierende transnationale Konstruktion europäischer Identität. Auf diese Weise werden Fragen danach, wo die Europäische Union beginnt und wo sie aufhört - also wo ihre Grenzen sind -, empirisch bearbeitbar. Dieser Ansatz führt über die politikwissenschaftliche und politisch-theoretische Diskussion über europäische Identität und die legitimen Grenzen Europas nicht zuletzt deswegen hinaus, weil er die Konstruktion von Identitäten und Grenzen in Europa nicht als ein reaktives, hochgradig bedingtes und immer erst zuletzt zu politisch-institutionellen Ordnungen hinzutretendes Element begreift, sondern solchen Konstruktionen eine transformatorische Kraft bereits im Prozess des Entstehens politischer Gemeinwesen zuschreibt.

Klaus Eder hat diesen Grundansatz in der Zwischenzeit - u. a. auf den Seiten des Berliner Journals - weiterentwickelt, indem er eine Kombination von Netzwerk- und Dis- 
kursanalyse vorschlägt, um den Prozessen der Konstitution europäischer Identität und soziokultureller Kohäsion in Europa auf die Spur zu kommen: „Gesellschaften halten zusammen, weil sie strukturelle Kopplungen (zwischen Systemen, Organisationen oder Individuen), also Netzwerkbeziehungen erzeugen. Und sie halten zusammen, weil sie über kollektiv geteilte Interpretationen der Realität verfügen, die von Sprache über Werte und Normen bis hin zu gemeinsam erzählten Geschichten reichen.“ (Eder 2007, S. 35). Diese beiden Dimensionen gesellschaftlicher Kohäsion stehen indes nicht einfach nebeneinander, sondern sind durch einen „Transzendierungseffekt" aufeinander bezogen. Er besteht darin, dass zweckorientierte Vergemeinschaftung - etwa Kooperation in sozialen Netzwerken - eine Eigenlegitimität annimmt, die nicht mehr am Output dieser Vergemeinschaftung gemessen werden muss, sondern sich aus unterstellten Grenzziehungen nach außen ergibt (ebd., S. 34). Nach diesem Modell hat sich die Idee der Nation weltweit durchgesetzt, woraus sich für Eder die empirische Frage ergibt, ob EU-Europa zu einer ähnlichen Transzendierung von Zweckgemeinschaft zu affektiv besetzter und umgrenzter Gemeinschaft in der Lage ist.

Dann jedoch gliedert Eder das Problem der Transzendierung ,,in die Frage nach der Emergenz transnationaler Kommunikationszusammenhänge und in die Frage der diskursiven Schließung dieses Kommunikationsraums" (ebd., S. 35) auf, das heißt in die Frage nach der Genese transnationaler sozialer Räume und Bewegungsmuster einerseits und Anzeichen für die Entstehung einer europäischen Erinnerungsgemeinschaft andererseits. Er untersucht jede dieser beiden Dimensionen für sich: die sozialen Netzwerke hinsichtlich der Zunahme ihrer Europäisierung und die Kollektivvorstellungen in Bezug auf die Kompatibilität oder Entgegensetzung nationaler und europäischer Erinnerungsrahmen. Man kann nun fragen, auf welche Weise diese beiden Dimensionen von Sozialität in Europa miteinander verknüpft sind, zum Beispiel, auf welchem Wege es zu einer Transzendierung der Zweckgemeinschaftlichkeit und ihrer Praktiken in Richtung einer Emergenz vorgestellter Gemeinschaftlichkeit in Diskursen kommt bzw. wie sich dies beobachten lässt. Anders gefragt: Welche kollektive Bedeutung, welche geteilten Vorstellungen, welcher Zweckorientierungen überschreitende Sinnhorizont sind in den Zweckbeziehungen bereits angelegt oder zumindest aktivierbar?

Der vorliegende Aufsatz setzt an dieser Stelle an. Er ist dem Versuch gewidmet, den „Transzendierungseffekt" nicht nur konzeptionell und hinsichtlich seiner Vorbedingungen zu entwerfen, sondern empirisch beobachtbar zu machen, das heißt, soziale Praktiken und Kollektivvorstellungen miteinander zu verknüpfen. Mit diesem Ziel wird im Folgenden eine praxistheoretische Orientierung vorgeschlagen. Codierungen von Grenzen des Kollektivsubjekts sind nicht nur in expliziten Diskurspraktiken, sondern auch in anderen Praktiken, wenn auch in nicht expliziter Weise, artikuliert, nämlich als Effekt ihrer Performanz (vgl. Reckwitz 2003). Die praxistheoretische Sicht kann so weit gehen, die „Bedeutung“ sozialer Realität als reinen Performanzeffekt alltäglicher, im Regelfall nicht weiter überdachter Handlungsvollzüge zu beschreiben: „social reality acquires its transcendent aura as a result of performative activities which are routinely misrecognized for what they are" (Pels 2002, S. 73).' Bedeutungen kollektiver Identität und ihrer Gren-

1 Dies stellt eine offensichtliche Fortführung ethnomethodologischer Positionen dar (vgl. für eine Zusammenfassung Mehan u. Wood 1975). 
zen gehen gemäß einem solchen Ansatz aus Praktiken hervor, die nicht primär - sei es intentional oder funktional - auf die Produktion solcher Bedeutungen ausgerichtet sind, sondern die auf alltäglicher, hochinstitutionalisierter Basis stattfinden. Beispiele hierfür wären etwa politisch ratifizierte Grenzregime (etwa das Schengen-Abkommen) und die dadurch ermöglichten Praktiken des ausweislosen Reisens, Praktiken der Internierung von Flüchtlingen („Flughafenasyl“, vgl. Bigo 2006) oder ökonomische (De-)Privilegierungen bestimmter Handelspartner, die alle bestimmte Eigen-Fremd-Vorstellungen „transportieren“ bzw. genauer: zur Emergenz bringen. Daher können nicht nur Symbolzirkulationsprozesse in den Massenmedien, sondern auch der transnationale Austausch von Personen(-gruppen) und Waren in der EU Implikationen und Resonanzen für Identitäten und Grenzen auf transnationaler Ebene haben.

Die Hauptfrage des vorliegenden Aufsatzes lautet somit: Wie wirken sich Praktiken und deren Arrangement in ökonomischen, sozialen und kulturellen Institutionen - etwa beim Austausch von Waren, Personen und Symbolen - inhaltlich auf die Artikulation von Identitäten und Grenzen in EU-Europa aus? Empirisch werden Praktiken kultureller Arbeit von Städtepartnerschaftsorganisationen untersucht. Städtepartnerschaften werden in der politikwissenschaftlichen Forschung zumeist als wichtige Bestandteile einer demokratischen, partizipatorischen und transnationalen politischen Kultur in Europa bezeichnet. Dieser Perspektive begegne ich mittels einer empirischen Analyse der Entstehung politischer Codierungen Europas in der konkreten, transnationalen Partnerschaftsarbeit, die hauptsächlich aus der Instituierung und Aufrechterhaltung von Zirkulationspraktiken besteht, die Symbole, Personen(-gruppen) und Waren involvieren. Zuvor muss indes die praxistheoretische Perspektive theoretisch weiter ausgeführt und auf die Frage symbolischer Grenzregime zugeschnitten werden.

\section{3 ,Social Imaginaries 6}

Wie bereits oben angedeutet, operieren die meisten Studien zu (Konstruktionen der) Identität und Grenzen Europas implizit oder explizit mit einer Methodologie, die die Voraussetzungen und die Entwicklung expliziter und zumeist öffentlicher Kommunikationen bzw. Semantiken untersucht. Typischerweise verwendete Begriffe sind „Diskurs“, „Narrativ" oder „Code“ (Diez 2001; Eisenstadt 1999; Giesen 1999, 2002; Rosamond 2001; Wodak u. Weiss 2002). Der unbestreitbare Vorteil dieser Studien liegt darin, dass sie anschlussfähig sowohl an historische wie auch an kulturwissenschaftliche (insbesondere literarische) Fragestellungen und Methodologien sind (vgl. etwa Brague 1993; Eisenstadt 2002; Kaelble 2002; Neumann 1999). Eine gewisse Einseitigkeit besteht indes darin, dass sie nur explizite Kommunikationen in die Analyse einbeziehen und Phänomene, die nicht als Kommunikationen entworfen sind, allenfalls als Rahmenbedingungen, nicht aber in ihrem je spezifischen Sinngehalt erfassen können. Mit anderen Worten: Der Sinn von Identität und Grenzen wird auf Diskurs bzw. Narrativ begrenzt; der Gedanke, dass auch Praktiken, die nicht als Sinngebungen angelegt sind, einen für Identitäten und Grenzen inhaltlich relevanten Sinn haben könnten, kann nicht aufkommen.

Dies ist anders bei Ansätzen, die, im Gegensatz zu rein diskursanalytisch vorgehenden Studien, sich für die Sinndimension von Praktiken interessieren. Der vorliegende Aufsatz 
bezieht sich daher auf Charles Taylor $(2002,2004)$ und andere, die unter Rückgriff auf Cornelius Castoriadis (1987) und Jacques Lacan (1978) den Begriff des „Imaginären“ in die Debatte zurückgebracht haben. Der theoretische Kernpunkt ist, dass imaginäre Bedeutung im Bereich des Politischen nicht „diskursiv konstruiert" wird, also nicht Produkt expliziter, auf die diskursive Sinngenese gerichteter Kommunikationspraktiken ist, sondern gleichsam als Nebenprodukt sozialer Praktiken entsteht. Das paradigmatische Beispiel bildet die Verbreitung der Idee der Nation im Europa des 18. und 19. Jahrhunderts. Sie ist nicht nur, wie etwa von Habermas (1962), Giesen (1991) und Anderson (1987) herausgearbeitet, Produkt einer gezielten intellektuellen Konstruktion kollektiver Identität, sondern liegt durch bestimmte Praktiken der Symbolzirkulation quasi von selbst nahe. Ein nationales Publikum bildete sich daher nicht (nur) als Folge einer expliziten Codierung, sondern war implizit bereits durch die Zirkulation der Schriften selbst vorausgesetzt. Die Tatsache, dass diese Schriften vorhanden und in Umlauf waren, beruhte auf der Unterstellung der Existenz eines nationalen Publikums. In dieser zirkulären Beziehung zwischen Kollektivvorstellung und den Praktiken der Symbolzirkulation liegt das imaginäre Moment. Die Nation erschien somit nicht nur als soziale Konstruktion im diskursiven Sinne, sondern als eine Vorstellung, deren Plausibilität wie von selbst den materiellen Praktiken der Publikation, Zirkulation, Verbreitung und Weitergabe entstieg.

Der Begriff der „Zirkulation“ ist dem Forschungsansatz des Imaginären also insofern anverwandt: Durch Zirkulation werden nicht nur materielle und immaterielle Güter distribuiert, sondern es wird unter den Empfängern auch die Vorstellung erzeugt, Teil eines größeren, abstrakten Zusammenhangs zu sein (vgl. Gaonkar u. Povinelli 2003). So können symbolische Zirkulationskontexte als Zusammenhänge bestimmt werden, die symbolischen „Mehrwert“ in der Form kollektiver Identitätsangebote hervorbringen. Lee u. LiPuma (2002) stellen in ihrer Lesart klassischer Studien zur Entstehung von Öffentlichkeit und nationaler Identität wie etwa derer von Jürgen Habermas (1962) und Benedict Anderson (1987) fest, dass die Konstruktion bürgerlich-nationaler Identität maßgeblich eine Folge von Zirkulationspraktiken kultureller Symbole gewesen ist. Durch den Umlauf literarischer und publizistischer Schriften unter einer Schicht von Bildungsbürgerinnen und -bürgern sei die Idee einer Einheit und kollektiven Handlungsform erzeugt worden, die die Einheiten der Zirkulationskette - die einzelnen lesenden und schreibenden Individuen - transzendiert. Die Vorstellung, es gebe so etwas wie „Gesellschaft" oder „die Nation", erscheint somit als kultureller Mehrwert der Symbolzirkulation zwischen Individuen und Institutionen. Dies geschieht durch einen doppelten Modus der Kollektivbeziehung derjenigen, unter denen Bedeutung im Umlauf ist: erstens vertikal auf den „Inhalt“ des Gesellschaftsentwurfs als symbolischen Mehrwert, zweitens lateral auf die Anderen, die der Vorstellung nach an der Symbolzirkulation beteiligt sind. Die Vorstellung eines „mehrwertigen“ Kollektivsubjekts mit eigenem Handlungsanspruch - der „Gesellschaft“, der „Nation“ oder durchaus auch der „EU“ - entsteht aus der Kombination vertikaler und lateraler Kollektivbezüge (Lee u. LiPuma 2002, S. 195 f.).

Die Spezifik dieses theoretischen Ansatzes zur Entstehung von Identitäten und Grenzziehungen kraft der Zirkulation von Symbolen lässt sich besonders plastisch herausstellen, vergleicht man sie mit der klassischen Studie zur politischen Kultur von Almond u. Verba (1963) über The civic culture. Gingen Almond und Verba davon aus, dass es miteinander vergleichbare, subjektiv verbürgte Einstellungsmuster und Dispositionen sind, die 
politische Kultur konstituieren und die sich vertikal auf „politische Objekte“ beziehen, wäre aus einer zirkulationstheoretischen Perspektive der Prozess der Konstruktion kollektiver Identität in Praktiken, die signifikante, laterale Andere voraussetzen, in den Blick zu nehmen. Vorstellungen nationaler Kollektive residieren daher nicht in Einstellungsmustern, sondern in vorausgesetzten Unterstellungen der Existenz nationaler Kollektive (vgl. Eder 2007), deren Alltagsessenzialiät (vgl. Pels 2002, S. 69) aus alltäglichen Praktiken der Symbolzirkulation wie Zeitungslektüre, Fernsehen oder Online-Surfen scheinbar selbstevident ist. Die Überzeugungskraft solcher symbolischen Gebilde beruht demnach auch nicht auf ihrer subjektiven oder sozialisatorischen Verbürgung, sondern auf ihrer Imaginarität als „faction“, d.h. als Amalgam von Fakt und Fiktion (ebd., S. 72).

Aus diesen Überlegungen lässt sich folgern, dass Zirkulationspraktiken nicht nur die Voraussetzung symbolischer Identifizierungen und Grenzziehungen bilden können, sondern - quasi als symbolischen „Mehrwert“ - Identitäten und Grenzen als imaginäre Größen hervorbringen. Mein theoretischer Ausgangspunkt ist somit, dass es empirisch beobachtbare Zirkulationspraktiken sind, die der sozialen Konstitution symbolischer Grenzregime zugrunde liegen. Zirkulationspraktiken erzeugen imaginäre Bedeutungen abstrakter „subjectivities“ (Gaonkar 2002, S. 5). Ihre Abstraktheit und Imaginarität besteht darin, dass ihre Realität das Produkt von Praktiken ist, welche wiederum jene imaginären Bedeutungen voraussetzen müssen, um funktionieren und Sinn haben zu können. Die Idee einer Nation etwa entsteigt gleichsam der Zirkulation von Symbolen in öffentlichen Massenmedien, während die Zurkenntnisnahme dieser Medien wiederum nur dann einen auch subjektiven Sinn ergibt, wenn die Existenz eines Massenpublikums, das doch unerfahrbar bleibt, als real vorausgesetzt werden kann.

In Bezug auf die Europäische Union und ihre symbolischen Grenzen stellt sich so die Frage, in welcher Weise Praktiken der Zirkulation von Personen, Gütern und Symbolen imaginäre Bedeutungen europäischer Grenzen aufrufen und konstituieren. Es geht daher nicht um den „Kontext“ von Grenzbedeutungen, sondern um die produktiven Kräfte, die den Praktiken Bedeutung entsteigen lassen. Solche Zirkulationspraktiken lassen sich am Beispiel von Städtepartnerschaften multidimensional untersuchen. Aus dieser theoretischen Sicht erscheinen Praktiken von Städtepartnerschaften als nicht als solche intendierten „Produktionsmittel“ imaginärer Kollektivbedeutung, das heißt europäischer Identität und europäischer Grenzen. Aus diesem Grund werden im empirischen Abschnitt sowohl inhaltliche als auch analytische und ethnografische Beschreibungen vorgelegt, die an den transnationalen Praktiken der Zirkulation von Waren und Personen zwischen verschiedenen, zu einer Partnerschaft zusammengeschlossenen Städten ansetzen, und im Hinblick auf ihre imaginäre, Grenzen ziehende Bedeutung befragt.

\section{Signifikanz von Städtepartnerschaften für eine Analyse imaginärer europäischer Grenzziehungen}

Zusätzlich zu der eben theoretisch geführten Argumentation bestehen mindestens zwei weitere Gründe, Städtepartnerschaften (SP) in Europa als überaus signifikant für die Praxis von Grenzkonstruktionen in Europa und deren politisch-kulturelle Bedeutung zu erachten. Mit diesen Gründen befasst sich der vorliegende Abschnitt. 
(a) Erstens wird die Geschichte der SP in Europa in der Forschung mit historisch einander folgenden Grenzregimen und deren Unterlaufen in Verbindung gebracht. Während - nach dieser Auffassung - direkt nach dem Zweiten Weltkrieg SP vor allem zwischen Frankreich und Deutschland die alten Gräben überbrücken helfen sollten und während der Blockkonfrontation europäischen Städten gar eine ,alternative Außenpolitik“ mit Städten in staatssozialistischen Ländern zugestanden oder angetragen wurde, sollten sie nach 1989 dazu beitragen, noch bestehende Klischees und „Grenzen in den Köpfen“ abzubauen. In den 1990er Jahren verschob sich der von der sozialwissenschaftlichen Literatur erwartete Integrationseffekt der SP von der (nun obsoleten) Problematik des Systemgegensatzes hin zum aufklärerischen Effekt, den die direkte Kommunikation zwischen europäischen Bürgern auf Augenhöhe zu haben versprach: Durch direkten Austausch, so die Hoffnung, könnten nach wie vor existierende nationale Stereotypen überwunden und die EU in ihrer Qualität als supranationales politisches Gebilde symbolisch gestärkt werden. Ihr integrativer Aspekt wurde so innerhalb unterschiedlicher historischer Konstellationen und in Bezug auf unterschiedliche gesellschaftliche Problematiken unterstrichen (vgl. Bock 1994; Mirek 1989, S. 37; Ullrich 1994; Wagner 1994).

In den vorhandenen sozialwissenschaftlichen Studien zu SP herrscht breiter Konsens darüber, dass diese transnationalen Bindungen zwischen Städten und Kommunen in hohem Maße zu kultureller Kohäsion, gegenseitigem Verstehen und der Erziehung zu bürgerschaftlichen Praktiken innerhalb Europas beitragen. Städtepartnerschaften werden als „Hoffnungsschimmer" einer auf breite Grundlage gestellten Demokratie betrachtet (Richter 1994, S. 36). Die Frage, wie der Erfolg von Partnerschaften zwischen Städten in Europa und jenseits einzuschätzen wäre, wird in dieser Forschung in Beziehung zu einer Art politischer Unmittelbarkeitserfahrung, zu politischer Mobilisierung und Aktivierung gesetzt. Diese Schwerpunktsetzung fällt besonders deutlich aus, wo es um europäische Integration geht. Der Städtepartnerschaftsbewegung wird höchste Bedeutung zugesprochen, ein Europa auf persönlicher Basis, ohne das der Gemeinsame Markt eine leere Hülle bleibe, ermöglicht zu haben (Mirek 1989, S. 41). Das Hauptargument war und ist, dass die Multiplizierung und internationale Diversifizierung institutioneller und persönlicher Bindungen, wie sie von den Städtepartnerschaftsorganisationen betrieben wurde, zur Auflösung von Klischees im europäischen Kontext und zur zivilgesellschaftlichen Mobilisierung für Europa als politisches Projekt beitragen könne (Mirek 1989; Wagner 1994; Wagner 1995, S. 17 ff.). Die normative Erwartung an die Partnerschaftsbewegung, ebenso wie das Selbstverständnis vieler der involvierten Akteure, kann somit in der Stärkung gegenseitigen Verstehens in Europa gesehen werden, ein Verstehen, das durch direkten Austausch von Personen und Erfahrungen und die Auflösung wechselseitiger Stereotypen in direkter Kommunikation ermöglicht werden soll. Sie stellen aus dieser Sicht ein Paradebeispiel für europäische Netzwerkbeziehungen dar (vgl. Eder 2007). Daher nimmt es nicht Wunder, dass sie für soziale Kräfte, die eine demokratische politische Kultur in Europa stärken, als beispielgebend angesehen werden (Kodolitsch 1989, S. 8 f., 14; Meyer 2004; Richter 1994).

Für den Ansatz des vorliegenden Artikels sind diese Einschätzungen vor allem deswegen interessant, weil sie die Leistung solcher Verständigungspraktiken stets in der Überwindung von Grenzen sehen, sei es in der unmittelbaren Nachkriegszeit die Überwindung 
der Barriere zwischen den ehemaligen Erzfeinden Deutschland und Frankreich, später der Ost-West-Konfrontation und seit den 1990er Jahren der „Grenzen in den Köpfen“, die in Europa immer noch existierten. Jenseits der normativ-theoretischen Herangehensweise, die den allergrößten Teil der existierenden Literatur zum Thema prägt, soll hier indes gefragt werden, welche Grenzüberwindungen, aber auch Grenzaufrichtungen die soziale Praxis der Städtepartnerschaftsarbeit tatsächlich zustande bringt. Es wird daher darum gehen, konkrete Praktiken zu untersuchen, in die Verständnisse von europäischer Identität und legitimen Grenzziehungen eingelagert sind.

(b) Städtepartnerschaften können auch deshalb als eine bevorzugte Empirie für die Erforschung von Grenzregimen in der Europäischen Union gelten, weil es sich bei ihnen um transnationale Milieus europäischer „Aktivbürgerschaft“ handelt. Bei Aktivbürgerschaft geht es um ein Konzept, das von Teilen der normativen politischen Theorie für die politische Integration Europas als zentral erachtet wird. So argumentiert Claudia Wiesner (2007, S. 17 ff.), dass das Ziel einer demokratischen Integration Europas durch vier Teilvorgänge begleitet werden muss: die Herausbildung einer politischen Öffentlichkeit, einer Zivilgesellschaft, einer demokratischen politischen Identität und einer Aktivbürgerschaft, das heißt einer Teilhabe der Bürgerinnen und Bürger am politischen Geschehen über die Teilnahme an Wahlen hinaus. Wiesner zufolge ist von diesen Teilprozessen derjenige der Bürgerschaft (zumindest in rechtlichem Sinne) nicht nur am weitesten fortgeschritten, sondern wirkt sich verursachend auf die anderen Teilprozesse aus. In Städtepartnerschaften nun lässt sich Aktivbürgerschaft als Praxis und in ihren Folgen empirisch beobachten, weil SP soziokulturelle Foren bürgerschaftlicher Aktivierung und politisch-integrationaler Anschlusskommunikation darstellen und damit auch auf das Problem der symbolischen Konstitution von Grenzen bezogen sind. Anders gesagt: In den Praktiken von SP zeigt sich Aktivbürgerschaft in Aktion und damit auch in ihren unintendierten Konsequenzen. Dieser Umstand macht es interessant, die politisch-kulturellen Auswirkungen der Praxis jener Aktivbürgerschaft - also die Emergenz der Bedeutung europäischer Identität und europäischer Grenzziehungen - einer Analyse zu unterziehen.

\section{Grenzen der Städtepartnerschaft: Gemeinschaft als gemeinsamer Markt}

Der empirische Abschnitt des Aufsatzes befasst sich, im Sinne einer Fallstudie, mit den Aktivitäten des Städtepartnerschaftsrings der hessischen Kleinstadt Braunfels. Es werden Ergebnisse teilnehmender Beobachtung sowie Analysen von Dokumenten vorgelegt. ${ }^{2} \mathrm{Die}$ öffentlichen Aktivitäten des Partnerschaftsrings sind vielgestaltig und erstrecken sich auf

2 Die Beobachtungen und Gespräche erfolgten zwischen 2002 und 2006 bei der Teilnahme an öffentlichen Veranstaltungen des Partnerschaftsrings hauptsächlich in Braunfels selbst, an denen ich in einigen Fällen teilnehmend-beobachtend beteiligt war, etwa beim Verkauf von Glühwein auf dem Braunfelser Weihnachtsmarkt. Die analysierten Dokumente sind erstens der Internetauftritt des Vereins, eine Vereinsbroschüre, die Präsentation des Vereins in der Selbstdarstellung der Stadt Braunfels sowie in einem Redemanuskript, das mir zur Verfügung gestellt wurde. An dieser Stelle möchte ich mich bei den Mitgliedern des Partnerschaftsrings herzlich für ihre Bereitschaft zum Gespräch und den Zugang zu den Dokumenten bedanken. Der vorliegende Abschnitt ist eine erweiterte Fassung der Analyse in Langenohl 2009. 
Felder wie Organisation von kulturellen Veranstaltungen und Sportfesten, Durchführung von Bildungsworkshops (meist zum Thema „Europa"), Delegations- und Bildungsreisen ins Ausland. Finanziert werden die Aktivitäten durch öffentliche Gelder, meist aus Mitteln der Europäischen Kommission, die je gesondert beantragt werden. Die Einwerbung von Mitteln ist eine Routineaktivität. Der Verein greift hierfür auch auf kostenlose Beratungsangebote zurück, um die Antragsarchitektur der EU zu meistern.

Die folgende Beschreibung nimmt an den Aktivitäten ihren Ausgang, die am sichtbarsten sind und mittels derer der Partnerschaftsring mit der Umwelt in Beziehung tritt. Entsprechend wird zunächst auf die Selbstpräsentation des Partnerschaftsrings (5.1), dann auf einen typischen Auftritt des Rings im sozialstrukturellen Rahmen der Kleinstadt (5.2) und schließlich auf einen ebenso typischen Auftritt einer Delegation des Rings in einer der europäischen Partnerstädte eingegangen (5.3).

\subsection{Die Selbstpräsentation}

Der Partnerschaftsring wurde 1968 gegründet, neun Jahre nachdem die Stadt Braunfels ihr erstes Partnerschaftsabkommen mit der französischen Stadt Bagnols-sur-Cèze unterzeichnet hatte. Der Verein versteht sich als eine Verbreiterung der Basis der Partnerschaftsidee nach der Schließung der ersten beiden Partnerschaftsabkommen: „Verantwortungsvolle Bürger waren sich jedoch auch bewusst, dass Städtepartnerschaften nur erfolgreich funktionieren können, wenn sie so im Bewusstsein der Bürger verankert sind, dass man sich besucht, ohne der direkten Hilfe der Verwaltung zu bedürfen. Bürger aller politischen Richtungen waren sich einig, dass Partnerschaften nur dann praktiziert werden können, wenn sie aus dem Rathaus heraus in die Bevölkerung hinein getragen werden. (...) An dem Gründungsabend (des Partnerschaftsrings) waren sich alle Anwesenden, darunter auch viele Jugendliche, einig, dass in Urkunden festgeschriebene Partnerschaften nur dann mit Leben erfüllt werden können, wenn sie nicht das Privileg einer bestimmten Gruppierung werden und wenn sie nicht nur durch Mandatsträger getragen werden." ${ }^{\text {* }}$

Der Partnerschaftsring stellt sich somit als eine integrale und inklusive Stütze des öffentlichen Lebens von Braunfels vor. In Gesprächen mit den Mitgliedern zeigte sich, dass die aktiven Mitglieder des Rings ganz überwiegend der Mittelschicht angehören - es sind unter ihnen Selbstständige, Lehrerinnen und Lehrer, Bankangestellte und Studierende -, die aber gleichzeitig zur ,guten Gesellschaft" des Ortes gehören, indem sie beispielsweise politische Ämter bekleiden oder mit der örtlichen politischen, wirtschaftlichen und adeligen Prominenz bekannt und befreundet sind. Auch in der offiziellen Selbstdarstellung der Stadt wird der Partnerschaftsring an prominenter Stelle erwähnt, indem herausgestellt wird, dass er vor einigen Jahren den Europäischen Preis für erfolgreiche Städtepartnerschaftsarbeit gewonnen hat, woran sich eine kurze Charakterisierung der sechs Partnerstädte in Belgien, Großbritannien, Frankreich, Österreich, Ungarn, Italien

3 http://www.partnerschaftsring-braunfels.de/geschichte, Zugriff am 13.11.2009, vier Rechtschreibfehler korrigiert. 
sowie in den USA anschließt. ${ }^{4}$ Auf diese Weise entsteht die Wahrnehmung eines (zumindest) europaweiten zwischenstädtischen, zivilgesellschaftlich institutionalisierten Netzwerkes, in dem Braunfels einen Knotenpunkt bildet.

\subsection{Der Markt}

Dem entspricht auch die Wahrnehmung des Partnerschaftsrings durch die städtische Öffentlichkeit. Der Verein ist daran beteiligt, Waren, Personengruppen und Symbole in internationalen Umlauf zu bringen, und setzt sich durch diese Drehscheibenfunktion mit dem städtischen Umfeld ins Verhältnis. Dies gilt zunächst für die Zirkulation von Waren. Der Ring führt Wein aus den französischen und italienischen Partnerstädten ein und verkauft ihn. Öffentlich geschieht dies auf dem alljährlich stattfindenden Weihnachtsmarkt. Der Anspruch besteht dabei darin, den „besten“ Glühwein, aus „französischem Wein“ hergestellt, zu verkaufen. Der Verkaufsstand befindet sich an einem zentralen Ort, nämlich auf dem historischen Marktplatz, während andere Glühweinstände eher an der Peripherie des Marktes liegen und die städtische Prominenz nicht in demselben Maße anziehen. Der flaschenweise Verkauf von Wein erfolgt hingegen auf einer nachfrageorientierten Basis, das heißt der Wein wird aus Frankreich und Italien nicht en gros importiert, sondern auf spezielle Anfragen hin auf den zahlreichen Auslandsreisen mit dem partnerschaftsringeigenen Kleinbus mitgebracht. Einerseits trägt der Weinverkauf also - mit Bourdieu (1990) gesprochen - zum Erhalt des Sozialkapitals des Vereins bei, das sich beispielhaft in den zahlreichen Besuchen der städtischen Prominenz am Glühweinstand artikuliert. Andererseits mehrt er das kulturelle Kapital der Abnehmer, die gerne auf die speziellen und individuellen Umstände des Weinerwerbs hinweisen, welche sich stark von generalisierten und anonymisierten Marktregeln abheben, weil sie in einen exklusiven und sozial spezifizierten Zirkulationskontext eingebunden sind. Auf diese Weise ist der Partnerschaftsring ein Element der städtischen Sozialstruktur und ihrer Vergleichsarrangements, indem er durch die exklusive, weil gegenüber dem Massenmarkt abgeschirmte Zirkulation von Waren den Tausch von sozialem gegen kulturelles Kapital ermöglicht.

Ähnliches gilt für die Zirkulation von Personen. Der Verein organisiert Reisen von Jugendlichen zu gemeinsamen Freizeiten, aber auch von örtlichen Sportlerinnen und Sportlern, die an regelmäßig stattfindenden, zwischen den Partnerstädten rotierenden Fußball- und Tennisturnieren teilnehmen. Weiterhin fahren regelmäßig Delegationen des Partnerschaftsrings zusammen mit örtlichen Politikern zu Veranstaltungen, die von den Partnerstädten veranstaltet werden. Der Grund solcher Reisen ist in der Regel außeralltäglicher Natur - es geht um Feier, Ritual und Repräsentation, sei es auf dem Feld traditioneller Festlichkeiten, im sportlichen Kontext oder bei kulturellen Veranstaltungen. Ähnlich wie bei der exklusiven Warenzirkulation entsteht auch hier der symbolische „Mehrwert" durch Exzeptionalität: Die Internationalität von Teilnehmenden an Veranstaltungen von ansonsten eher lokaler Bedeutung - seien dies nun Stadtteilfeste, Tennis-

4 http://www.braunfels.com/sites/gensite.asp?SID=cms271120091244441759302\&Art=033:44 $\& C=1$, Zugriff am 26.11.2009. Diese Charakterisierung findet sich auch im Webauftritt des Vereins, vgl. http://www.partnerschaftsring-braunfels.de/unsere-partnerstaedte (Zugriff am 13.11.2009). 
turniere oder Konzerttage - hebt diese gegenüber regional vergleichbaren hervor und verleiht ihnen ein kosmopolitisches Flair. Umgekehrt entsteht bei den Angereisten der Eindruck, jenseits des üblichen Massentourismus an authentischen öffentlichen Events teilzunehmen, und sie werden in die Lage versetzt, gegenüber „gewöhnlichen“ Touristen privilegierte Präferenzen und Meinungen auszubilden und zu artikulieren.

Dieser auf ethnografischen Beschreibungen beruhende Bericht macht den transnationalen Charakter der Zirkulationspraktiken, mithilfe derer sich der Partnerschaftsring mit der sozialen und kulturellen Ökonomie von Braunfels ins Benehmen setzt, deutlich. Ebenso wie der Tausch von kulturellem und sozialem Kapital nur aufgrund von Prestigesymbolen funktioniert (Wein), deren Exklusivität auf ihrer ebenso internationalen wie individualisierten Importgeschichte beruht, nährt sich das Flair von Veranstaltungen ansonsten eher lokaler Bedeutung zu einem Großteil von der internationalen Anreise von Gästen, die zwischen den Städtepartnerschaftsorganisationen zirkulieren. Im Zusammenhang des vorliegenden Forschungsinteresses muss nun gefragt werden, wie diese Ergebnisse zu Grenzkonstruktionen (in) der Europäischen Union in Verbindung gesetzt werden können, das heißt wie ihr innerer Zusammenhang mit Artikulationen von Identitäten und Grenzen (in) der EU beschaffen ist. Es wurde gezeigt, dass die Kulturarbeit der Partnerstädte in hohem Maße mit ihren internationalen Praktiken der Warenzirkulation und der internationalen Aufspannung einer Prestigeökonomie verquickt ist. Dies stellt den Ausgangspunkt für ein Verstehen der imaginären Komponente von Identitäts- und Grenzentwürfen dar, die in der Partnerschaftsarbeit immer wieder auch explizit artikuliert werden. Einem solchen Entwurf wendet sich der nächste Unterabschnitt zu.

\subsection{Die Festrede}

Oben wurde argumentiert, dass der Austausch von Personen und Waren als Teil der Aktivitäten des Braunfelser Partnerschaftsrings einen Austausch von kulturellem gegen soziales Kapital und umgekehrt einrichtet, indem die getauschten Personen und Waren als international, exklusiv und authentisch etikettiert werden. In diesem Unterabschnitt wird auf die imaginäre Bedeutung des aus diesen Zirkulationsverhältnissen hervorgehenden Mehrwerts eingegangen. Dazu wenden wir uns nun einer Analyse eines Kultur-Events in der italienischen Partnerstadt Feltre zu, der im Jahre 2005 stattfand, aber aufgrund seines wiederkehrenden Charakters als typisch gelten kann. Es handelt sich um einen „palio“, das heißt um ein jährlich begangenes traditionelles Fest, organisiert von den Vierteln der jeweiligen Stadt. Diese präsentieren sich als Teile der Stadt und gleichzeitig als miteinander wetteifernde Gemeinden, was Ausdruck in stark ritualisierten Sportwettkämpfen findet (etwa Kunstreiten oder Fahnenschwenken). Gäste aus Braunfels und den anderen Partnerstädten werden zum palio nicht nur regelmäßig eingeladen, sondern sind aktiv an ihm beteiligt - typischerweise in Form von Reden, die durch politische oder sozial-kulturelle Repräsentanten der Partnerstädte gehalten werden. Im Jahre 2005 etwa hielt der stellvertretende Bürgermeister von Braunfels eine Rede, in der er an zentraler Stelle auf die damals bevorstehende Erweiterungswelle der Europäischen Union und Zukunftsszenarien zu sprechen kam.

Die Rede beginnt mit einem Rückblick auf die eigene Reiseerfahrung des nun über 60Jährigen in Westeuropa. Aus dieser Perspektive wird die Osterweiterung der EU zunächst 
als positiv dargestellt, aber sogleich mit durch die EU auferlegten ökonomischen Konditionen in Verbindung gebracht. Die Länder Tschechien, Polen und Ungarn werden in diesem Kontext als solche herausgestellt, die diese EU-Konditionen (bisher) erfüllt haben und insofern eine optimistische Zukunftsperspektive eröffnen. Daran schließt sich ein Kata$\log$ politisch umzusetzender Forderungen an die neuen Mitgliedsstaaten an, die sich vor allem auf die Vereinheitlichung von Umweltstandards, Grenzkontrollen und die befürchtete Abwanderung von Arbeitsplätzen in die Beitrittsländer beziehen. Mit Sorge wird die (damals) nächste mögliche Erweiterungswelle betrachtet (genannt werden Bulgarien, Rumänien und die Türkei), weil bei diesen Ländern allgemein eine weite Entfernung von EU-Standards ausgemacht wird. Die Rede endet mit einem Appell, die „Grenzen in den Köpfen" abzubauen, und analogisiert die Offenheit der europäischen Grenzen durch eine Aufzählung verschiedener europäischer, nationaltypischer Genussgüter: „Lassen Sie uns miteinander mit allen 25 Mitgliedsstaaten dafür sorgen, dass die französischen Weine, die italienische Küche, der niederländische Käse, die belgischen Pralinen, die spanischen Oliven, die ungarische Salami, das tschechische Bier und die anderen feinen Dinge, die uns Genuss bescheren, schon einmal die Barrieren von europäischen Grenzen in unseren Köpfen verschwinden lassen."

Das Vergleichsarrangement, das in dieser Rede konstruiert wird, ist ein chronologisches, nämlich zwischen den alten, den neuen und den potenziell zukünftigen EU-Staaten, wobei die „alte“ EU als unproblematisch normsetzend erscheint. Die Forderungen an die neuen Mitgliedsstaaten verweisen auf die Zirkulation von Waren, die mögliche Umverteilung von Arbeitsplätzen und die Diffusion von politischen, ökonomischen und ökologischen Normen. Die administrative und ökonomische Sichtweise bestimmt auch die Forderungen und die Kritik an Staaten zukünftiger Erweiterungswellen, weil diese auf absehbare Zeit nicht reif für die EU seien. Der schließende Appell an den Abbau der „Grenzen in den Köpfen" annulliert das Trennende zwischen alten und neuen EU-Staaten und verstärkt gleichzeitig den symbolischen Ausschluss der potenziell zukünftigen Mitglieder. Einerseits wird das Trennende der ökonomischen Sichtweise durch die Vorstellung der Zirkulation und des gemeinsamen Genusses hochwertiger Waren aufgehoben, andererseits werden aber nur Waren genannt, die für die Staaten der damaligen EU typisch waren. Der Appell zum kognitiven Grenzabbau zwischen alten und neuen Mitgliedsstaaten auf der Grundlage heraufbeschworener Warenzirkulation geht somit mit der Ausgrenzung zukünftiger Mitgliedsstaaten vom gemeinsamen Mahl einher.

Bringt man die ethnografischen mit den inhaltsanalytischen Ergebnissen zusammen, ergibt sich das Bild einer kulturellen Ökonomie, in der einerseits der soziale Status der Mitglieder des Partnerschaftsrings im sozialstrukturellen Feld der beteiligten Städte gesichert und reproduziert wird und andererseits - etwa auf Festreden in den Partnerstädten - ein Bild europäischer Integration gezeichnet wird, das stark mit Metaphern des Essens und der Kommensalität operiert. Im geschilderten Beispiel steht der Vergleich von alten, neuen und potenziell zukünftigen Mitgliedsstaaten im Zusammenhang mit der durch Warenzirkulation ermöglichten Akkumulation von Sozial- und kulturellem Kapital und mit der symbolischen Aufwertung lokaler Veranstaltungen und der Teilnahme an diesen.

5 Fuchs, Hans Werner (2005). Speech at the meeting „Perspectives and problems about the new countries joining the European Union". Feltre: Ms. 
Kommensalität, die internationale Zirkulation von Konsumgütern, ihr Beitrag zu einer lokalen Sozialstruktur und die öffentliche Darstellung der EU als (Lebensmittel-)Markt im Rahmen von Städtepartnerschaften verdichten sich so zu einem Imaginären europäischer Integration, das den Markt in den Vordergrund rückt. Erstens wird dem Markt diskursiv (in der Rede) eine hohe und normativ anspruchsvolle Bedeutung beigemessen. Zweitens hat dieser Markt reale sozialstrukturelle Konsequenzen, weil er an der Aufrechterhaltung lokaler Prestigeökonomien beteiligt ist (die Internationalität der zirkulierten Waren und Personen). Drittens kann die Metapher des europäischen gemeinsamen Mahles zum Vehikel symbolischer Grenzziehung genau deswegen werden, weil sie die Existenz eines freien, durch keine politische Barriere behinderten Tauschs von Lebensmitteln in der EU als selbstverständlich voraussetzen kann.

Die diskursive Metapher des (Lebensmittel-)Marktes, die in der Rede die abstrakte EU-Kollektivität bezeichnet, verfängt somit genau deswegen, weil ein solcher Markt, über Praktiken der Zirkulation von Waren, Personen und Symbolen in der EU, an höchst konkrete sozialstrukturelle und Prestigeökonomien angeschlossen ist (s. 5.2). Die Aufstufung dieser in der Praxis implizit bleibenden Bedeutung des Marktes zu einer diskursiven Metapher, die Selbstevidenz beansprucht - also der „Transzendierungseffekt“ (Eder 2007, s. o.) - ist der Kern des imaginären Geschehens. Die oft geäußerten Kritiken, dass die EU nichts als ein Markt sei, werden in dieser Transzendierung gleichsam umgewendet: Was die EU zu einem Gemeinwesen macht und sie nach außen abgrenzt, ist gerade ihre Marktförmigkeit. Diese imaginäre Gleichsetzung der Grenzen von Markt und Gemeinwesen, die sich diskursiv als Metapher der Kommensalität auf der Grundlage der Zirkulation von Speisen ausbildet, ist mit einer sozialen, kulturellen und Warenökonomie verschränkt, die jene Gleichsetzung immer schon voraussetzt.

\section{Praktiken des Tauschs und die imaginären Grenzen der EU}

Das theoretische Hauptargument dieses Aufsatzes lautet, dass politische Identitäten und Grenzen sich in Prozessen des Umlaufs von Personen(-gruppen), Waren und Symbolen bilden, die nicht auf die Konstruktion von Identität und Grenzen hin entworfen sind. Stattdessen erzeugen sie aufgrund ihrer praxeologischen Merkmale, zum Beispiel ihrer Anschlüsse an ökonomische und sozialstrukturelle Prozesse, imaginäre abstrakte Kollektivitäten. Städtepartnerschaften, die dem Anspruch nach der Durchführung ,kultureller" Aktivitäten und Kooperationen innerhalb der Europäischen Union dienen, belegen diesen imaginären Prozess, in dem Verständnisse des Politischen nicht-politischen Prozessen entsteigen. Die Signifikanz und Bedeutsamkeit symbolischer Konstruktionen transnationaler Kollektivität in der EU, wie sie etwa in offiziellen Ansprachen wie der hier analysierten Festrede anzutreffen sind, ergibt sich erst aus ihrer Einbettung in interund transnationale Umläufe von Waren und Personen(-gruppen), in denen soziales, ökonomisches und kulturelles Kapital zirkuliert. Die diskursiv explizierten und die imaginär implizierten Repräsentationen der Europäischen Union als abstrakter Kollektivität formen ein Bild der EU als ein politisches Kollektiv gerade kraft ihrer Marktförmigkeit. Die imaginären Grenzen dieses Gebildes bildet die (Nicht-)Zulassung zu besagten Zirkulationsprozessen. 
Die große Bedeutung der Metapher und Praxis des Marktes weist auf eine für viele gegenwärtige Gesellschaften charakteristische Tendenz hin, Bürgerschaft („citizenship“) in Begriffen von Konsumverhalten („,consumership“) umzusetzen (vgl. für einen Überblick Lamla 2010). Symbolische Grenzziehungen, die auf soziale Praktiken zurückgehen, entstehen somit in einem universalisierten Konsumentenmarktmodell. In diesem Modell manifestiert sich die imaginäre Bedeutung politischer Integration und Grenzziehung weniger als Verschmelzung von Bürger und Bourgeois, sondern als Amalgamierung von Bürger und Konsument.

In welchem Sinne sind aber diese imaginären Grenzen, wie sie sich am Beispiel von Städtepartnerschaften aus praxeologischer Perspektive darstellen lassen, spezifisch europäisch? Dies wird sichtbar, wenn man in Betracht zieht, dass die Grenzziehungen, die durch am Markt als Praxis und Metapher orientierte Aktivitäten von Städtepartnerschaftsorganisationen hervorgebracht werden, in einer Kontinuität mit dem politischen Projekt der Europäischen Union stehen. Insofern sie auf kulturelle Ökonomien sowie die Zirkulation von Waren und Personen orientiert sind, beziehen sie sich auf ein Paradigma politischer Integration, das der Ökonomie eine hohe Dignität zugesteht und die EU als Ganze in ihrer Entstehung historisch geprägt hat - die Montanunion war ein internationales Wirtschaftsabkommen, das Kriege verhindern sollte - und noch prägt.

Spezifisch europäisch wäre an dieser imaginären politischen Identität also ihre Verankerung in der Geschichte. Während im Falle nationalstaatlich imaginierter politischer Identität die, um es so zu nennen, bürgerschaftliche Aktivkonsumentenschaft grell mit der (angeblichen) Geborgenheit nationalgesellschaftlicher Gemeinschaftlichkeit kontrastiert, kann sich der Bürger-Konsument in der Europäischen Union in einem emphatischen Sinne auf Gründungsdokumente ihrer historischen Vorläufer (nämlich der verschiedenen EGs und der Montanunion) berufen. Dies bedeutet aber, dass auch Vertragsverhältnisse - denn der Markt ist nichts anderes als ein Makromodell von Vertraglichkeit -, denen zumeist zweckrationale Motivationsformen nachgesagt werden, die nicht über das je konkrete Vertragsverhältnis hinausgehen (vgl. Eder 2007, aber auch Axelrod 1987), zum Ausgangspunkt und Vehikel emphatischer Kollektivität werden können.

Wenn daher auch die Verbindung von Markt als Zirkulationssphäre und imaginären Repräsentationen politischer Kollektivität bereits bei der Entstehung der nationalen Idee eine Rolle spielte, wie Taylor $(2002,2004)$ und andere argumentieren, unterscheidet sich das EU-Imaginäre darin, dass es eine offenkundige Verwirklichungsform der Allianz von Marktförmigkeit und abstrakter Kollektivität ist (vgl. hierzu Berting 1995). ${ }^{6}$ Vielleicht gehen daher die Vorwürfe, die EU sei bloß ein Markt, an ihrer historischen Bedeutung vorbei, stellt sich die Europäische Union doch als erste reflexive Instanz der Verbindung von Markt und Kollektivität dar. Darin ist sie das bislang radikalste - und höchst selbstbewusste - politische Projekt in der Episteme der Moderne.

6 Dies wirft ein interessantes Licht auf Vertrags- und Vorvertragstheorien gesellschaftlicher Integration. Denn aus der Sicht des Imaginären und seines Hervorgehens aus Praktiken der warenförmigen Zirkulation wäre zu sagen, dass „Gesellschaftsvertrag“ und „Kollektivbewusstsein“ nicht einander, je nach theoretischer Präferenz, vorausgehen, sondern zusammen entstehen. 


\section{Literatur}

Almond, G., \& Verba, S. (1963). The civic culture: Political attitudes in five nations. Princeton: Princeton University Press.

Anderson, B. (1987). Imagined communities: Reflections on the origin and spread of nationalism. London: Verso.

Axelrod, R. M. (1987). Die Evolution der Kooperation. München: Oldenbourg.

Beck, U., \& Delanty, G. (2006). Europe from a cosmopolitan perspective. In G. Delanty (Hrsg.), Europe and Asia beyond east and west (S. 11-23). London: Routledge.

Berting, J. (1995). Patterns of exclusion: Imaginaries of class, nation, ethnicity and gender in Europe. In J. N. Pieterse \& B. Parekh (Hrsg.), The decolonization of imagination: Culture, knowledge and power (S. 149-165). London: Zed Books.

Bigo, D. (2006). Globalized-in-security: The field and the ban-opticon. In N. Sakai \& J. Solomon (Hrsg.), Translation, biopolitics, colonial difference (S. 109-155). Hong Kong: Hong Kong University Press.

Bock, H. M. (1994). Europa von unten. Zu den Ursprüngen und Anfängen der deutsch-französischen Gemeindepartnerschaften. In A. Jünemann, E. Richter, \& H. Ullrich (Hrsg.), Gemeindepartnerschaften im Umbruch Europas (S. 13-35). Frankfurt a.M.: Peter Lang.

Böckenförde, E. W. (1997). Welchen Weg geht Europa? München: Siemens-Stiftung.

Brague, R. (1993). Europa. Eine exzentrische Identität. Frankfurt a.M.: Campus.

Bourdieu, P. (1990). Social space and symbolic power. In P. Bourdieu (Hrsg.), In other words: Essays toward a reflexive sociology (S. 123-139). Cambridge: Polity Press.

Castoriadis, C. (1987). The imaginary institution of society. Cambridge: Polity Press.

Delanty, G. (2006). Introduction: The idea of a post-western Europe. In G. Delanty (Hrsg.), Europe and Asia beyond east and west (S. 1-7). London: Routledge.

Diez, T. (2001). Speaking „Europe"? The politics of integration discourse. In T. Christiansen, K. E. Jorgensen, \& A. Wiener (Hrsg.), The social construction of Europe (S. 85-100). London: Sage.

Eder, K. (2003). Öffentlichkeit und Demokratie. In M. Jachtenfuchs \& B. Kohler-Koch (Hrsg.), Europäische Integration (S. 85-120). Opladen: Leske + Budrich.

Eder, K. (2007). Europa als besonderer Kommunikationsraum: Zur Frage der sozialen Integration einer kulturell heterogenen Gemeinschaft. Berliner Journal für Soziologie, 17, 33-50.

Eder, K., Kantner, C., \& Trenz, H.-J. (2000). Transnationale Öffentlichkeit und die Strukturierung politischer Kommunikation in Europa. Antrag auf Förderung eines Forschungsvorhabens im Rahmen des DFG-Schwerpunkts „Regieren in Europa“, Berlin 2000. http://www2.rz.hu-berlin.de/struktur/Forschung/transoeff/Euro-DFG-Antrag.pdf. Zugegriffen Juli 2007.

Eisenstadt, S. N. (1999). Kollektive Identitätskonstruktionen in Europa, den Vereinigten Staaten, Lateinamerika und Japan. Eine vergleichende Betrachtung. In R. Viehoff \& R. T. Segers (Hrsg.), Kultur, Identität, Europa. Über die Schwierigkeiten und Möglichkeiten einer Konstruktion (S. 370-400). Frankfurt a.M.: Suhrkamp.

Eisenstadt, S. N. (2002). Some observations on multiple modernities. In S. N. Eisenstadt, D. Sachsenmaier, \& J. Riedel (Hrsg.), Reflections on multiple modernities: European, Chinese and other interpretations (S. 27-41). Leiden: Brill.

Fuchs, D., \& Klingemann, H.-D. (2000). Eastward enlargement and the identity of Europe. Discussion Paper FS III 00-206. Berlin: Wissenschaftszentrum Berlin.

Gaonkar, D. P. (2002). Toward new imaginaries. An introduction. Public Culture, 14, 1-19.

Gaonkar, D. P., \& Povinelli, E. A. (2003). Technologies of public forms: Circulation, transfiguration, recognition. Public Culture, 15, 385-397.

Gerhards, J. (2004). Europäische Werte: Passt die Türkei kulturell zu Europa? Aus Politik und Zeitgeschichte, B 38/2004, 14-20.

Giesen, B. (1991). Die Intellektuellen und die Nation: Eine deutsche Achsenzeit. Frankfurt a.M.: Suhrkamp. 
Giesen, B. (1999). Europa als Konstruktion der Intellektuellen. In R. Viehoff \& R. T. Segers (Hrsg.), Kultur, Identität, Europa. Über die Schwierigkeiten und Möglichkeiten einer Konstruktion (S. 130-146). Frankfurt a.M.: Suhrkamp.

Giesen, B. (2002). Constitutional practice or community of memory? Some remarks on the collective identity of Europe. In D. Sachsenmaier, S. N. Eisenstadt, \& J. Riedel (Hrsg.), Reflections on multiple modernities: European, Chinese and other interpretations (S. 193-213). Leiden: Brill.

Greven, M. T. (1998). Mitgliedschaft, Grenzen und öffentlicher Raum. Problemdimensionen der Demokratisierung der Europäischen Union. In B. Kohler-Koch (Hrsg.), Regieren in entgrenzten Räumen (S. 249-270). Opladen: Westdeutscher Verlag.

Habermas, J. (1962). Strukturwandel der Öffentlichkeit. Untersuchungen zu einer Kategorie der bürgerlichen Gesellschaft. Neuwied: Luchterhand.

Habermas, J. (1998). Die postnationale Konstellation. Frankfurt a.M.: Suhrkamp.

Habermas, J. (2001). Braucht Europa eine Verfassung? In J. Habermas (Hrsg.), Zeit der Übergänge. Kleine politische Schriften IX (S. 85-103). Frankfurt a.M.: Suhrkamp.

Immerfall, S., \& Sobisch, A. (1997). Europäische Integration und europäische Identität. Aus Politik und Zeitgeschichte, B 10/97, 25-37.

Kaelble, H. (2002). Constitutional practice or community of memory? Some remarks on the collective identity of Europe. In D. Sachsenmaier, S. N. Eisenstadt, \& J. Riedel (Hrsg.), Reflections on multiple modernities: European, Chinese and other interpretations (S. 193-213). Leiden: Brill.

Kielmansegg, P. G. (1996). Integration und Demokratie. In M. Jachtenfuchs \& B. Kohler-Koch (Hrsg.), Europäische Integration (S. 47-72). Opladen: Leske + Budrich.

Kodolitsch, P. v. (1989). Einleitung. In P. v. Kodolitsch (Hrsg.), Kommunale „Außenpolitik“. Zur Auslandsarbeit der Gemeinden und zu den innerdeutschen Städtepartnerschaften (S. 7-18). Berlin: Deutsches Institut für Urbanistik.

Krossa, A. S. (2009). Conceptualizing European society on non-normative grounds: Logics of sociation, glocalization and conflict. European Journal of Social Theory, 12, 249-264.

Lacan, J. (1978). Die vier Grundbegriffe der Psychoanalyse. Das Seminar von Jacques Lacan, Bd. 11. Olten: Walter-Verlag.

Lamla, J. (2010). Konsumkulturen im Kapitalismus. Vier theoretische Perspektiven auf die Verbraucherdemokratie. In H.-G. Soeffner (Hrsg.), Unsichere Zeiten. Herausforderungen gesellschaftlicher Transformation. Verhandlungen des 34. Kongresses der DGS in Jena 2008. CD-ROM. Wiesbaden: VS Verlag für Sozialwissenschaften (i. E.).

Langenohl, A. (2009). The imagination of Europe in twin town activities: Rethinking political culture in the European Union. In M. Albert et al. (Hrsg.), Transnational political spaces (S. 212-236). Frankfurt a.M./New York: Campus.

Lee, B., \& LiPuma E. (2002). Cultures of circulation: the imaginations of modernity. Public Culture, 14, 191-213.

Lepsius, M. R. (1999). Die Europäische Union. Ökonomisch-politische Integration und kulturelle Pluralität. In R. Viehoff \& R. T. Segers (Hrsg.), Kultur - Identität - Europa. Über die Schwierigkeiten und Möglichkeiten einer Konstruktion (S. 201-222). Frankfurt a.M.: Suhrkamp.

Mehan, H., \& Wood H. (1975). The reality of ethnomethodology. New York: Wiley.

Meyer, T. (2004). Die Identität Europas. Der EU eine Seele? Frankfurt a.M.: Suhrkamp.

Mirek, H. (1989). Die Entwicklung der Städtepartnerschaften. In P. v. Kodolitsch (Hrsg.), Kommunale „Außenpolitik“. Zur Auslandsarbeit der Gemeinden und zu den innerdeutschen Städtepartnerschaften (S. 33-46). Berlin: Deutsches Institut für Urbanistik.

Neumann, I. (1999). Uses of the other: „, The East" in European identity formation. Minneapolis: University of Minnesota Press.

Pels, D. (2002). Everyday essentialism: Social inertia and the „Münchhausen effect“. Theory, Culture \& Society, 19, 69-89. 
Reckwitz, A. (2003). Grundelemente einer Theorie sozialer Praktiken. Eine sozialtheoretische Perspektive. Zeitschrift für Soziologie, 32, 282-301.

Reese-Schäfer, W. (1999). Supranationale oder transnationale Identität. Zwei Modelle kultureller Integration in Europa. In R. Viehoff \& R. T. Segers (Hrsg.), Kultur - Identität - Europa. Über die Schwierigkeiten und Möglichkeiten einer Konstruktion (S. 253-266). Frankfurt a.M.: Suhrkamp.

Richter, E. (1994). Die Gemeinde als Basis europäischer Integration - Subsidiarität und Bürgernähe. In A. Jünemann, E. Richter, \& H. Ullrich (Hrsg.), Gemeindepartnerschaften im Umbruch Europas (S. 37-55). Frankfurt a.M.: Peter Lang.

Rosamond, B. (2001). Discourses of Globalization and European Integration. In T. Christiansen, K. E. Jørgensen, \& A. Wiener (Hrsg.), The social construction of Europe (S. 158-175). London: Sage.

Soysa, I. de, \& Zervakis, P. (Hrsg.). (2002). Does culture matter? The relevance of culture in politics and governance in the Euro-Mediterranean zone. Discussion paper C 111. Bonn: Zentrum für Europäische Integrationsforschung.

Steeg, M. v. de (2003). Bedingungen für die Entstehung von Öffentlichkeit in der EU. In A. Klein et al. (Hrsg.), Bürgerschaft, Öffentlichkeit und Demokratie in Europa (S. 169-190). Opladen: Leske + Budrich.

Taylor, C. (2002). Modern social imaginaries. Public Culture, 14, 91-124.

Taylor, C. (2004). Modern social imaginaries. Durham: Duke University Press.

Trenz, H.-J. (2001). Modes of self-description of a European society. From , unity in diversity“ to the „diversity of units“. Paper for the 5th Conference of the European Sociological Association, Helsinki, 28.8.-1.9.2001.

Trenz, H.-J., Klein, L., \& Koopmans, R. (2003). Demokratie-, Öffentlichkeits- und Identitätsdefizite in der EU. Diagnose und Therapiefähigkeit. In A. Klein et al. (Hrsg.), Bürgerschaft, Öffentlichkeit und Demokratie in Europa (S. 7-13). Opladen: Leske + Budrich.

Ullrich, H. (1994). Vorwort. In A. Jünemann, E. Richter, \& H. Ullrich (Hrsg.), Gemeindepartnerschaften im Umbruch Europas (S. 7-11). Frankfurt a.M.: Peter Lang.

Vobruba, G. (2008). Die Entwicklung der Europasoziologie aus der Differenz national/europäisch. Berliner Journal für Soziologie, 18, 32-51.

Wagner, B. (1994). Städtepartnerschaften zwischen Ost- und Westeuropa. Eine Analyse am Beispiel bundesdeutscher Städte und Gemeinden. In A. Jünemann, E. Richter, \& H. Ullrich (Hrsg.), Gemeindepartnerschaften im Umbruch Europas (S. 109-130). Frankfurt a.M.: Peter Lang.

Wagner, B. (1995). Partnerschaften deutscher Städte und Gemeinden: Transnationale Beiträge zur internationalen Sicherheit. Münster: Lit.

Wiesner, C. (2007). Bürgerschaft und Demokratie in der Europäischen Union. Berlin: Lit.

Wodak, R., \& Weiss, G. (2002). The roles of political, cultural and linguistic ideologies in the discursive construction of European identities: Organizing, representing and legitimizing Europe. LAUD papers, Series A, No. 562. Essen: Linguistic Agency.

PD Dr. Andreas Langenohl, geb. 1970. PD Dr., Leiter der Forschungsgruppe „Idiome der Gesellschaftsanalyse“ am Exzellenzcluster 16 „Kulturelle Grundlagen von Integration“ der Universität Konstanz. Forschungsschwerpunkte: Finanzmarkt- und Wirtschaftssoziologie, gesellschaftliche Transformation, Europasoziologie, Modernisierungs- als Kulturtheorie, kollektive Erinnerung, Epistemologie der Sozialwissenschaften. Ausgewählte Veröffentlichungen: How to change other people's institutions: Discursive entrepreneurship and the boundary object of competition/competitiveness in the German banking sector. In: Economy and Society 37, 2008; Tradition und Gesellschaftskritik. Eine Rekonstruktion der Modernisierungstheorie, 2007; Finanzmarkt und Temporalität. Imaginäre Zeit und die kulturelle Repräsentation der Gesellschaft (= Qualitative Soziologie, Bd. 7), 2007. 